\title{
ON ROBUST EXPONENTIAL STABILITY OF A CLASS OF ATTRACTOR NEURAL NETWORKS *
}

\author{
Sun Changyin ${ }^{1,3} \quad$ Zhang Shi $^{2}$ \\ ${ }^{1}$ College of Electrical Engineering, Hohai University, Nanjing, 210098, China \\ ${ }^{2}$ College of Automation, Nanjing University of Technology, Nanjing, 210009, China \\ ${ }^{3}$ Research Institute of Automation, Southeast University, Nanjing, 210096, China
}

\begin{abstract}
Robust Exponential stability of continuous-time attractor neural networks with delays is discussed. A new sufficient condition ensuring existence and uniqueness of periodic solution for a general class of interval dynamical systems are obtained. Discretetime analogue of the continuous-time systems with periodic input is formulated and we study their dynamical characteristics. The robust exponential stability and periodicity of the continuous-time systems is preserved by the discrete-time analogue without any restriction imposed on the uniform discretization step-size. Copyright (C) 2005 IFAC
\end{abstract}

Keywords: Periodic solution, robust, discrete-time analogue, stability.

\section{INTRODUCTION}

The properties of equilibrium points of neural systems play an important role in some practical problems, such as optimization solvers (Chen, 2000; Forti, 1995; Kennedy, 1988; Tank and Hopfield, 1986; Sudharsanan and Sundareshan, 1991), pattern recognition (Liu and Lu, 1997) and image compression (Venetianer and Roska, 1998). It is well known that an equilibrium point can be viewed as a special periodic solution of continuous-time neural systems with arbitrary period (Zhang, 2002; Sun and Feng, 2003, 2004;). In this sense the analysis of periodic solutions of neural systems may be considered to be more general sense than that of equilibrium points. In addition, the existence of periodic solutions of continuous-time neural networks is an interesting dynamic behavior. It has been found applications in learning theory (Townley, 2000), which is motivated by the fact that learning process usually requires repetition. On the other hand, the dynamical characteristics of neural networks may often be destroyed by its unavoidable uncertainty due to the existence of modeling errors, external disturbance and parameter fluctuation during the implementation on very-large-scale-integration (VLSI) chips. Thus, it is important to investigate the

*This work was supported by China Postdoctoral Science Foundation and Jiangsu Planned Projects for Postdoctoral Research Funds. periodicity and robustness of the network against such errors and fluctuation. In order to overcome this difficulty, (Liao, 1998; Arik, 2003; and Sun, 2003) have extended the model of delayed Hopfield neural networks to interval-delayed neural systems. We further extend the model of delayed Hopfield neural networks with constant input to interval dynamical systems with periodic input in (Sun, 2004).

Dynamical analysis of continuous-time attractor neural networks with delays have been the new world-wide focus (Van and Zou, 1998; Cao, 2000; Gopalsamy, 1994; Liao and Wang, 2003; Sun, Zhang et al, 2002; Mohamad, 2001). However, in implementing the continuous-time neural system for simulation or computational purposes, it is essential to formulate a discrete-time system which is an analogue of the continuous-time system. A method which is commonly used in the formulation of a discrete-time analogue is by discretizing the continuous-time system. Certainly, the discrete-time analogue when derived as a numerical approximation of continuous-time system is desired to preserve the dynamical characteristics of the continuous-time system. Once this is established, the discrete-time analogue can be used without loss of functional similarity to the continuous-time system and preserving any physical or biological reality that the continuous-time system has. Though there exist a lot of numerical schemes (such as Euler scheme, RungeKutta scheme) that can be used to obtain discrete- 
time analogues of continuous-time neural networks. However, it is generally known that these numerical schemes can exhibit spurious equilibrium and spurious asymptotic behavior, which are not present in the continuous-time counterparts. The existence of spurious equilibrium and asymptotic behavior in a discrete-time analogue can happen if one holds the positive discretization step size fixed and let the original parameters (i.e. parameters of the continuous-time system) vary within the asymptotic parameter space of the continuous-time systems. As a consequence, one has to impose limitations either on the size of $h$ or on the original parameters so as to avoid the existence of spurious equilibrium and spurious asymptotic behavior in the discrete-time analogues. With such limitations, the computational capability of the continuous-time network will not be achieved fully by the discrete-time analogues. Here, we refer to (Mohamad and Gopalsamy, 2003; Sun and Feng, 2005) and their references therein for related discussions on the importance of discretetime counterparts.

In this paper, we will derive a easily checkable condition to guarantee robust exponential periodicity of continuous-time attractor neural networks with delays for pattern recognition. We will also study the robust exponential periodicity of discrete-time neural systems with delays. To the best knowledge of ours, this is the first time to investigate the robust exponential periodicity of discrete-time neural networks with delays.

\section{PROBLEM STATEMENT}

Consider the model of continuous-time neural network with delays described by the following functional differential equations

$$
\begin{gathered}
\dot{x}_{i}(t)=-d_{i} x_{i}(t)+\sum_{j=1}^{n} a_{i j} f_{j}\left(x_{j}\left(t-\tau_{j}\right)\right)+I_{i}(t), t \geq 0 \\
x_{i}(t)=\phi_{i}(t), i=1,2, \cdots, n,
\end{gathered}
$$

where $0 \leq \tau_{j} \leq \tau$ is the transmission time delay of the $j$ th unit , $x_{i}(t)$ is the state vector of the $i$ th unit at time $t, a_{i j}, b_{i j}$ are constants. For simplicity, let $D$ be an $n \times n$ constant diagonal matrix with diagonal elements $d_{i}>0, \quad i=1,2, \ldots, n, A=\left(a_{i j}\right)$ is $n \times n$ constant interconnection matrix, $f_{j}\left(x_{j}\left(t-\tau_{j}\right)\right)$ denotes the activation function of the $j$ th unit at time $t-\tau_{j}, I(t)=\left(I_{1}(t), I_{2}(t), ., I_{n}(t)\right)^{T} \in \mathrm{R}^{n}$ is an input periodic vector function with period $\omega$, i.e., there exists a constant $\omega>0$ such that $I_{i}(t+\omega)=I_{i}(t) \quad(i=1,2, \cdots, n)$ for all $t \geq 0$, and $x=\left(x_{1}, x_{2}, \ldots, x_{n}\right)^{T} \in \mathrm{R}^{n}$. Suppose further $f \in \mathrm{GLC}$ in $\mathrm{R}^{n}$, i.e., for each $j \in\{1,2, \cdots, n\}, f_{j}: \mathrm{R} \rightarrow \mathrm{R}$ is globally Lipschitz continuous with Lipschitz constant
$M_{j} \geq 0$, that is, $\left|f_{j}(u)-f_{j}(v)\right| \leq M_{j}|u-v|$ for all $u, v \in \mathrm{R}$. Moreover, we will use norms of vector $x$ in $\mathrm{R}^{n}$, which is defined as $\|x\|=\sum_{i=1}^{n}\left|x_{i}\right|$.

As a special case of neural system (1), the delayed neural networks with constant input vector $I=\left(I_{1}, I_{2}, \ldots, I_{n}\right)^{T} \in \mathrm{R}^{n}$ have been studied widely by many researchers. This system is described by the following functional differential equations

$$
\begin{gathered}
\dot{x}_{i}(t)=-d_{i} x_{i}(t)+\sum_{j=1}^{n} a_{i j} f_{j}\left(x_{j}\left(t-\tau_{j}\right)\right)+I_{i}, t \geq 0 \\
x_{i}(t)=\phi_{i}(t),-\tau \leq t \leq 0, i=1,2, \cdots, n,
\end{gathered}
$$

Define $x_{t}(\theta)=x(t+\theta), \theta \in[-\tau, 0], t \geq 0$. Let

$$
\left\|x_{t}\right\|=\sup _{-\tau \leq \theta \leq 0} \sum_{i=1}^{n}\left|x_{i}(t+\theta)\right|,
$$

Let $x^{*}=\left(x_{1}^{*}, x_{2}^{*}, \cdots, x_{n}^{*}\right)$ be the equilibrium point of system (2), i.e.,

$$
d_{i} x_{i}^{*}=\sum_{j=1}^{n} a_{i j} f_{j}\left(x_{i}^{*}\right)+I_{i}
$$

In practice implementation of neural networks, the value of the constants $d_{i}$ and connection weight coefficients $a_{i j}$ depend on certain resistance and capacitance value which are subject to uncertainties. This may lead to some deviations in the value of $d_{i}$, and $a_{i j}$. The deviations and perturbations of the neuron charging time constants and the weights of interconnections are bounded in general. In the following, we correspondingly intervalize the above mentioned quantities by defining the set of matrices as follows similar to (Liao, 1998; Sun and Feng, 2003).

$$
\begin{gathered}
D_{I}=\left\{D=\operatorname{diag}\left(d_{i}\right)_{n \times n}: \underline{D} \leq D \leq \bar{D},\right. \\
\left.\quad \text { i.e., } \underline{d}_{i} \leq d_{i} \leq \bar{d}_{i}, i=1,2, \ldots, n\right\} ; \\
A_{I}=\left\{A=\left(a_{i j}\right)_{n \times n}: \underline{A} \leq A \leq \bar{A},\right. \\
\text { i.e., } \left.\underline{a}_{i j} \leq a_{i j} \leq \bar{a}_{i j}, i, j=1,2, \ldots, n\right\} .
\end{gathered}
$$

In the following, we formulate a discrete-time analogue of the continuous-time neural networks (1) by a semi-discretization technique. For detail, reader can refer to (Sun and Feng, 2004).

$$
\begin{aligned}
x_{i}(m+1) & =x(m) e^{-d_{i} h} \\
+ & \theta_{i}(h)\left\{\sum_{j=1}^{n} a_{i j} f_{j}\left(x_{j}\left(m-\sigma_{j}\right)\right)+I_{i}(m)\right\}
\end{aligned}
$$

for $i=1,2, \cdots, n, m \in Z_{0}^{+}$, where

$$
\theta_{i}(h)=\frac{1-e^{-d_{i} h}}{d_{i}}, i=1,2, \cdots, n .
$$

It can be verified that $\theta_{i}(h)>0$ if $d_{i}>0, h>0$. System (4) is our discrete-time analogue of the continuous-time system (1). It is easily to verify that the discrete-time analogue (4) converges towards the continuous-time network (1) when $h \rightarrow 0^{+}$. 
In order to study the discrete-time analogue (3), we assume that the system (3) is supplemented with an initial condition, a consequence of the discretization, of the form $x_{i}(l)=\varphi_{i}(l)$ for $l \in[-\sigma, 0]_{z}$ where $i=1,2, \cdots, n, \sigma=\max _{j=1,2, \cdots, n}\left\{\sigma_{j}\right\}$, and the external input function are periodic function with positive integer period $\varpi$, i.e.,

$$
I_{i}(m+\varpi)=I_{i}(m), i=1,2, \cdots, n, m \in Z^{+} .
$$

As a special case of neural system (4), the delayed discrete-time neural networks with constant input vector $I=\left(I_{1}, I_{2}, \ldots, I_{n}\right)^{T} \in \mathrm{R}^{n}$ is described by the following functional differential equations

$$
\begin{aligned}
x_{i}(m+1) & =x(m) e^{-d_{i} h} \\
+ & \theta_{i}(h)\left\{\sum_{j=1}^{n} a_{i j} f_{j}\left(x_{j}\left(m-\sigma_{j}\right)\right)+I_{i}\right\} .
\end{aligned}
$$

Let $x^{*}=\left(x_{1}^{*}, x_{2}^{*}, \cdots, x_{n}^{*}\right)^{T}$ denote an equilibrium point of (5). We obtain from (5) that

$$
x_{i}^{*}=x_{i}^{*} e^{-d_{i} h}+\theta_{i}(h)\left\{\sum_{j=1}^{n} a_{i j} f_{j}\left(x_{j}^{*}\right)+I_{i}\right\} .
$$

from which one easily obtains the equilibrium point equation (3) of system (2). Obviously, the discretetime analogue (5) preserves the equilibrium $x^{*}$ of the continuous-time network (2).

\section{MAIN RESULTS}

In this section, we will present the results concerning the robust exponential periodicity of interval delayed neural networks of the form (1) and (3) for each

$$
D=\operatorname{diag}\left(d_{i}\right)_{n \times n} \in D_{I}, \quad A=\left(a_{i j}\right)_{n \times n} \in A_{I} \text {. }
$$

Theorem 1: Suppose $f \in$ GLC. If there exists a positive diagonal matrix $\Lambda=\operatorname{diag}\left(\lambda_{1}, \lambda_{2}, \ldots, \lambda_{n}\right)$ such that the system parameter $d_{i}, a_{i j}(i, j=1,2, \cdots, n)$ satisfy the following conditions

$$
\lambda_{i} \underline{d}_{i}-M_{i} \sum_{j=1}^{n} \lambda_{j}\left|a_{j i}^{*}\right|>0 \text {, for } i=1, \cdots, n,
$$

where

$$
\left|a_{i j}^{*}\right|=\max _{1 \leq i, j \leq n}\left\{\left|\underline{a}_{i j}\right|,\left|\bar{a}_{i j}\right|\right\},
$$

then for every periodic input $I(t)$, the delayed neural system (1) is robustly exponentially periodic.

Proof. Let $C=C\left([-\tau, 0], \mathrm{R}^{n}\right)$ be the Banach space of all continuous function from $[-\tau, 0]$ to $\mathrm{R}^{n}$ with the topology of uniform convergence. For any $\phi \in C$, let $\|\phi\|=\sup _{-\tau \leq t \leq 0} \sum_{i=1}^{n}\left|\phi_{i}(t)\right|$. Given any $\phi, \varphi \in C$, let $\quad x(t, \phi)=\left(x_{1}(t, \phi), x_{2}(t, \phi), \cdots, x_{n}(t, \phi)\right)^{T} \quad$ and $x(t, \varphi)=\left(x_{1}(t, \varphi), x_{2}(t, \varphi), \cdots, x_{n}(t, \varphi)\right)^{T} \quad$ be the solutions of (1) starting from $\phi$ and $\varphi$ respectively.

Define $\quad x_{t}(\phi)=x(t+\theta, \phi), \quad \theta \in[-\tau, 0], \quad$ then $x_{t}(\phi) \in C$ for all $t \geq 0$. Thus, it follows from (1) that

$$
\begin{aligned}
& \dot{x}_{i}(t, \phi)-\dot{x}_{i}(t, \varphi)=-d_{i}\left(x_{i}(t, \phi)-x_{i}(t, \varphi)\right) \\
& +\sum_{j=1}^{n} a_{i j}\left(f_{j}\left(x_{j}\left(t-\tau_{j}, \phi\right)\right)-f_{j}\left(x_{j}\left(t-\tau_{j}, \varphi\right)\right)\right)
\end{aligned}
$$

for $t \geq 0, i=1,2, \cdots, n$.

We consider function $F_{i}(\cdot)$ defined by

$$
F_{i}\left(\varepsilon_{i}\right)=\lambda_{i}\left(\underline{d}_{i}-\varepsilon_{i}\right)-M_{i} e^{\varepsilon_{i} \tau} \sum_{j=1}^{n} \lambda_{j}\left|a_{j i}^{*}\right|
$$$$
\text { for } \varepsilon_{i} \in[0, \infty), i=1, \cdots, n \text {. }
$$

We have from (4) and (6) that $F_{i}(0)>0$ for $i=1, \cdots, n$ and hence by the continuity of $F_{i}(\cdot)$ on $[0, \infty)$, there exists a constant $\varepsilon>0$ such that

$$
\begin{gathered}
F_{i}(\varepsilon)=\lambda_{i}\left(\underline{d}_{i}-\varepsilon\right)-M_{i} e^{\varepsilon \tau} \sum_{j=1}^{n} \lambda_{j}\left|a_{j i}^{*}\right|>0, \\
\text { for } i=1, \cdots, n
\end{gathered}
$$

We will use the following positive definite Lyapunov function candidate defined as follows:

$$
\begin{gathered}
V(t)=\sum_{i=1}^{n} \lambda_{i}\left(\left|x_{i}(t, \phi)-x_{i}(t, \varphi)\right| e^{\varepsilon t}\right. \\
\left.+\sum_{j=1}^{n}\left|a_{i j}\right| M_{j} \int_{t-\tau_{j}}^{t}\left|x_{j}(s, \phi)-x_{j}(s, \varphi)\right| \times e^{\varepsilon\left(s+\tau_{j}\right)} d s\right) .
\end{gathered}
$$

Define the upper right derivative of $V(t)$ by

$$
D^{+} V(t)=\lim _{h \rightarrow 0^{+}} \sup [V(t+h)-V(t)] / h .
$$

Computing the upper right derivative of $V(t)$ along (5) for $t \geq 0$, we have

$$
\begin{aligned}
& D^{\dagger} V(t)=\sum_{i=1}^{n} \lambda_{i} \operatorname{sgn}\left(x_{i}(t, \phi)-x_{i}(t, \varphi)\right)^{[}-\left(d_{i} x_{i}(t, \phi)-d_{i} x_{i}(t, \varphi)\right) \\
& +\sum_{j=1}^{n} a_{i j}\left(f_{j}\left(x_{j}\left(t-\tau_{j}, \phi\right)-f_{j}\left(x_{j}\left(t-\tau_{j}, \varphi\right)\right)\right] e^{\varepsilon t}\right. \\
& +\sum_{=1}^{n} \lambda_{i}\left|x_{i}(t, \phi)-x_{i}(t, \varphi)\right| \varepsilon e^{\varepsilon t} \\
& +\sum_{i=1}^{n} \sum_{j=1}^{n} \lambda_{i}\left|a_{i j}\right| M_{j}\left(\left|x_{j}(t, \phi)-x_{j}(t, \varphi)\right| e^{\varepsilon\left(t+\tau_{j}\right)}\right. \\
& \left.-\left|x_{j}\left(t-\tau_{j}, \phi\right)-x_{j}\left(t-\tau_{j}, \varphi\right)\right| e^{\varepsilon t}\right) \\
& \leq \sum_{i=1}^{n} \lambda_{i}\left[-d_{i}\left|x_{i}(t, \phi)-x_{i}(t, \varphi)\right|\right. \\
& \left.+\sum_{j=1}^{n}\left|a_{i j} \| f_{j}\left(x_{j}\left(t-\tau_{j}, \phi\right)\right)-f_{j}\left(x_{j}\left(t-\tau_{j}, \varphi\right)\right)\right|\right] e^{\varepsilon t} \\
& +\sum_{=1}^{n} \lambda_{i}\left|x_{i}(t, \phi)-x_{i}(t, \varphi)\right| \varepsilon e^{\varepsilon t} \\
& +\sum_{i=1}^{n} \sum_{j=1}^{n} \lambda_{i}\left|a_{i j}\right| M_{j}\left(\left|x_{j}(t, \phi)-x_{j}(t, \varphi)\right| e^{\varepsilon\left(t+\tau_{j}\right)}\right. \\
& \left.-\left|x_{j}\left(t-\tau_{j}, \phi\right)-x_{j}\left(t-\tau_{j}, \varphi\right)\right| e^{\varepsilon t}\right) \\
& \leq \sum_{i=1}^{n} \lambda_{i}\left[-d_{i}\left|x_{i}(t, \phi)-x_{i}(t, \varphi)\right|\right] e^{\varepsilon t} \\
& +\sum_{=1}^{n} \lambda_{i}\left|x_{i}(t, \phi)-x_{i}(t, \varphi)\right| \varepsilon e^{\varepsilon t} \\
& +\sum_{i=1}^{n} \sum_{j=1}^{n} \lambda_{i}\left|a_{i j}\right| M_{j}\left(\left|x_{j}(t, \phi)-x_{j}(t, \varphi)\right| e^{\varepsilon\left(t+\tau_{j}\right)}\right) \\
& \leq-\sum_{i=1}^{n}\left\{\lambda_{i}\left(\underline{d}_{i}-\varepsilon\right)-M_{i} e^{\varepsilon \tau} \sum_{j=1}^{n} \lambda_{j}\left|a_{j i}^{*}\right|\right\}\left|x_{i}(t, \phi)-x_{i}(t, \varphi)\right| e^{\varepsilon t} \\
& \leq 0 \text {. }
\end{aligned}
$$


Therefore,

$$
V(t) \leq V(0), t \geq 0 .
$$

From (8), we obtain

$$
e^{\varepsilon t}\left(\min _{1 \leq i \leq n} \lambda_{i}\right) \sum_{i=1}^{n}\left|x_{i}(t, \phi)-x_{i}(t, \varphi)\right| \leq V(t)
$$

and

$$
\begin{aligned}
& V(0)=\sum_{i=1}^{n} \lambda_{i}\left[\left|x_{i}(0, \phi)-x_{i}(0, \varphi)\right|\right. \\
& \left.\quad+\sum_{j=1}^{n}\left|a_{i j}\right| M_{j} \int_{-\tau_{j}}^{0}\left|x_{j}(s, \phi)-x_{j}(s, \varphi)\right| e^{\varepsilon\left(s+\tau_{j}\right)} d s\right] \\
& \leq\left|\max _{1 \leq i \leq n} \lambda_{i}+\bar{M} \tau e^{\varepsilon \tau} \sum_{i=1}^{n} \lambda_{i} \max _{1 \leq j \leq n}\left(\left|a_{i j}^{*}\right|\right)\right||| \phi-\varphi \|,
\end{aligned}
$$

where $\bar{M}=\max _{1 \leq i \leq n}\left\{M_{i}\right\}$ is constant. Therefore, from (9), we get

$$
\sum_{i=1}^{n}\left|x_{i}(t, \phi)-x_{i}(t, \varphi)\right| \leq \alpha\|\phi-\varphi\| \exp (-\varepsilon t)
$$

for all $t \geq 0$, where

$$
\alpha=\frac{\max _{1 \leq i \leq n} \lambda_{i}+\bar{M} \tau e^{\varepsilon \tau} \sum_{i=1}^{n} \lambda_{i} \max _{1 \leq j \leq n}\left(\left|a_{i j}^{*}\right|\right)}{\min _{1 \leq i \leq n} \lambda_{i}} \geq 1 .
$$

It follows from (10) that

$$
\left\|x_{t}(\phi)-x_{t}(\varphi)\right\| \leq \alpha\|\phi-\varphi\| \exp (-\varepsilon(t-\tau))
$$

We can choose a positive integer $m$ such that

$$
\alpha \exp (-\varepsilon(m \omega-\tau)) \leq \frac{1}{4} .
$$

Define a Poincaré mapping $H: C \rightarrow C$ by $H \phi=x_{\omega}(\phi)$. Then from (11), we can derive that

$$
\left\|H^{m} \phi-H^{m} \varphi\right\| \leq \frac{1}{4}\|\phi-\varphi\|,
$$

where $H^{m} \phi=x_{m \omega}(\phi)$. This implies that $H^{m}$ is a contraction mapping. Therefore, there exists a unique fixed point $\phi^{*} \in C$ such that $H^{m} \phi^{*}=\phi^{*}$. So, $H^{m}\left(H \phi^{*}\right)=H\left(H^{m} \phi^{*}\right)=H \phi^{*}$. This shows that $H \phi^{*} \in C$ is also a fixed point of $H^{m}$, hence, $H \phi^{*}=\phi^{*}$, that is, $x_{\omega}\left(\phi^{*}\right)=\phi^{*}$. Let $x\left(t, \phi^{*}\right)$ be the solution of (1) through $\left(0, \phi^{*}\right)$. By using $I(t+\omega)=I(t)$ for $t \geq 0, x\left(t+\omega, \phi^{*}\right)$ is also a solution of (1). Note that $x_{t+\omega}\left(\phi^{*}\right)=x_{t}\left(x_{\omega}\left(\phi^{*}\right)\right)=x_{t}\left(\phi^{*}\right)$ for $t \geq 0$, then $x\left(t+\omega, \phi^{*}\right)=x\left(t, \phi^{*}\right)$ for $t \geq 0$. This shows that $x\left(t, \phi^{*}\right)$ is a periodic solution of (1) with period $\omega$. From (11), it is easy to see that all other solutions of (1) converge to this periodic solution exponentially as $t \rightarrow+\infty$.

Remark 1. When $I=\left(I_{1}, I_{2}, \cdots, I_{n}\right)$ is a constant vector, then for any constant $T \geq 0$ we have $I=I(t+T)=I(t)$ for $t \geq 0$. Thus by the results obtained, when the sufficient condition in Theorem 1 is satisfied, a unique periodic solution becomes a periodic solution with any positive constant as its period. So, the periodic solution reduced to a constant solution, that is, an equilibrium point. Furthermore, all other solutions globally exponentially converge to this equilibrium point as $t \rightarrow+\infty$. The unique equilibrium point of the delayed neural system (2) is globally robustly exponentially stable. Obviously, the results obtained are consistent with the exponential stability results that were recently reported in (Cao, 2000; Sun, et al, 2002) and (Sun and Feng, 2003; Zhang, 2002).

Theorem 2: Suppose $f \in$ GLC and the positive stepsize is fixed. If there exists a positive diagonal matrix $\Lambda=\operatorname{diag}\left(\lambda_{1}, \lambda_{2}, \ldots, \lambda_{n}\right)$ such that the system parameter $d_{i}, a_{i j}(i, j=1,2, \cdots, n)$ satisfy the following conditions

$$
\lambda_{i} \underline{d}_{i}-M_{i} \sum_{j=1}^{n} \lambda_{j}\left|a_{j i}^{*}\right|>0, \quad \text { for } i=1, \cdots, n,
$$

where

$$
\left|a_{i j}^{*}\right|=\max _{1 \leq i, j \leq n}\left\{\underline{a}_{i j}|,| \bar{a}_{i j} \mid\right\},
$$

then for every periodic input $I(m)$, the delayed neural system (3) is robustly exponentially periodic.

Proof. Let $B=B\left([-k, 0]_{Z}, \mathrm{R}^{n}\right)$ be the Banach space with norm $\|\phi\|=\max _{l \in[-k, 0]_{Z}}\left(\sum_{i=1}^{n}\left|\phi_{i}(l)\right|\right)$ for any $\phi \in B$. Given any $\phi, \psi \in B$, let $x(m, \phi)=\left(x_{1}(m, \phi), x_{2}(m, \phi), \cdots, x_{n}(m, \phi)\right)^{T} \quad$ and $x(m, \psi)=\left(x_{1}(m, \psi), x_{2}(m, \psi), \cdots, x_{n}(m, \psi)\right)^{T}$ be the solutions of (16) starting from $\phi$ and $\psi$ respectively. Define $\quad x^{m}(\phi)(l)=x(m+l, \phi), \quad l \in[-\sigma, 0]_{z}, \quad$ then $x^{m}(\phi)(l) \in B$ for all $m \in Z^{+}$. Thus, it follows from (3) that

$$
\begin{gathered}
\left|x_{i}(m+1, \phi)-x_{i}(m+1, \psi)\right| \leq\left|x(m, \phi)-x_{i}(m, \psi)\right| e^{-d_{i} h} \\
+\theta_{i}(h)\left\{\sum_{j=1}^{n}\left|a_{i j} M_{j}\left(x_{j}\left(m-\sigma_{j}, \phi\right)-x_{j}\left(m-\sigma_{j}, \psi\right)\right)\right|\right\} \\
\text { for } i=1,2, \cdots, n .
\end{gathered}
$$

We consider function $\tilde{F}_{i}(\cdot)$ defined by

$$
\tilde{F}_{i}\left(r_{i}\right)=\lambda_{i}\left(1-r_{i} e^{-d_{i} h}\right)-M_{i} \theta_{i}(h) \sum_{j=1}^{n} \lambda_{j}\left|a_{j i}^{*}\right| r_{i}^{\sigma_{i}+1}
$$$$
\text { for } r_{i} \in[1, \infty), i=1, \cdots, n \text {. }
$$

We have from (4) and (14) that $\tilde{F}_{i}(1) \geq \theta_{i}(h)\left(\lambda_{i} \underline{d}_{i}-M_{i} \sum_{j=1}^{n} \lambda_{j}\left|a_{j i}^{*}\right|\right)>0$ for $i=1, \cdots, n$ and hence by the continuity of $\tilde{F}_{i}(\cdot)$ on $[1, \infty)$, there exists a constant $r>1$ such that

$$
\begin{gathered}
\tilde{F}_{i}(r)=\lambda_{i}\left(1-r e^{-d_{i} h}\right)-M_{i} \theta_{i}(h) \sum_{j=1}^{n} \lambda_{j}\left|a_{j i}^{*}\right| r^{\sigma_{i}+1} \geq 0, \\
\text { for } i=1, \cdots, n
\end{gathered}
$$

In the following, we consider nonnegative sequence $z_{i}(m)$ defined by

$$
\begin{array}{r}
z_{i}(m)=\lambda_{i}^{-1} r^{m} \frac{\left|x_{i}(m, \phi)-x_{i}(m, \psi)\right|}{\theta_{i}(h)} \\
\text { for } i=1, \cdots, n, m \in[-\sigma, \infty)_{Z} .
\end{array}
$$


By using (18) and (21) we obtain the following inequalities:

$$
\begin{aligned}
& z_{i}(m+1)=\lambda_{i}^{-1} r^{m+1} \frac{\left|x_{i}(m+1, \phi)-x_{i}(m+1, \psi)\right|}{\phi_{i}(h)} \\
& \leq r e^{-d_{i} h} z_{i}(m)+\lambda_{i}^{-1} \sum_{j=1}^{n}\left|a_{i j}\right| \lambda_{j} M_{j} \theta_{j}(h) r^{\sigma_{j}+1} z_{j}\left(m-\sigma_{j}\right), \\
& \text { for } i=1, \cdots, n, m \in[-\sigma, \infty)_{z} .
\end{aligned}
$$

Accordingly, we will use the following positive definite Lyapunov function candidate:

$$
V(m)=\sum_{i=1}^{n}\left(z_{i}(m)+\lambda_{j}^{-1} \sum_{j=1}^{n}\left|a_{i j}\right| \lambda_{j} M_{j} \theta_{j}(h) r^{k_{j}+1} \sum_{l=m-\sigma_{j}}^{m-1} z_{j}(l)\right),
$$

$$
\text { for } m \in Z_{0}^{+} \text {. }
$$

Calculating the difference $\Delta V(m)=V(m+1)-V(m)$ along (22), we obtain

$$
\begin{aligned}
\Delta V(m) \leq & \sum_{i=1}^{n}\left\{r e^{-d_{i} h} z_{i}(m)\right. \\
& +\lambda_{i}^{-1} r \sum_{j=1}^{n}\left|a_{i j}\right| \lambda_{j} M_{j} \theta_{j}(h) r^{\sigma_{j}+1} z_{j}\left(m-\sigma_{j}\right) \\
+ & \lambda_{i}^{-1} \sum_{j=1}^{n}\left|a_{i j}\right| \lambda_{j} M_{j} \theta_{j}(h) r^{\sigma_{j}+1} \sum_{l=m+1-k_{j}}^{m} z_{j}(l)-z_{i}(m) \\
- & \left.\lambda_{i}^{-1} \sum_{j=1}^{n}\left|a_{i j}\right| M_{j} \lambda_{j} \theta_{j}(h) r^{\sigma_{j}+1} \sum_{l=m-k_{j}}^{m-1} z_{j}(l)\right\} \\
= & -\sum_{i=1}^{n}\left\{1-r e^{-d_{i} h}-M_{i} \theta_{i}(h) \sum_{j=1}^{n} \lambda_{i}^{-1} \lambda_{j}\left|a_{j i}\right| r^{\sigma_{i}+1}\right\} z_{i}(m) \\
= & -\sum_{i=1}^{n} \lambda_{i}^{-1}\left\{\lambda_{i}\left(1-r e^{-d_{i} h}\right)-M_{i} \theta_{i}(h) \sum_{j=1}^{n} \lambda_{j}\left|a_{j i}\right| r^{\sigma_{i}+1}\right\} z_{i}(m) \\
\leq & -\sum_{i=1}^{n} \lambda_{t}^{-1}\left\{\lambda_{i}\left(1-r e^{-d_{i} h}\right)-M_{i} \theta_{i}(h) \sum_{j=1}^{n} \lambda_{j}\left|a_{j i}^{*}\right| r^{\sigma_{i}+1}\right\} z_{i}(m)
\end{aligned}
$$

for $m \in Z_{0}^{+}$. By using (20) in (24) we assert that $\Delta V(m) \leq 0 \quad$ for $\quad m \in Z_{0}^{+} \quad$ which implies $V(m) \leq V(0)$ for $m \in Z^{+}$. It then follows from (19) that

$$
\begin{aligned}
\sum_{i=1}^{n} z_{i}(m) & \leq \sum_{i=1}^{n}\left\{z_{i}(0)\right. \\
& \left.+\lambda_{i}^{-1} \sum_{j=1}^{n}\left|a_{i j}\right| \lambda_{j} M_{j} \theta_{j}(h) r^{\sigma_{j}+1} \sum_{l=-\sigma_{j}}^{-1} z_{j}(l)\right\}
\end{aligned}
$$

and by substituting (21) in the above we obtain

$$
\begin{aligned}
\sum_{i=1}^{n} \lambda_{i}^{-1} \frac{\left|x_{i}(m, \phi)-x_{i}(m, \psi)\right|}{\theta_{i}(h)} \leq\left(\frac{1}{r}\right)^{m} \\
\quad \times \sum_{i=1}^{n} \lambda_{i}^{-1}\left\{1+M_{i} \theta_{i}(h) \lambda_{j} \sum_{j=1}^{n}\left|a_{j i}\right| r^{\sigma_{i}+1} \sigma_{i}\right\} \\
\times\left(\sup _{l \in[-\sigma, 0]_{z}} \frac{\left|\phi_{i}-\psi_{i}\right|}{\theta_{i}(h)}\right)
\end{aligned}
$$

with the implication

$$
\frac{\lambda_{\min }}{\theta_{\max }(h)} \sum_{i=1}^{n}\left|x_{i}(m, \phi)-x_{i}(m, \psi)\right| \leq \frac{\lambda_{\max }}{\theta_{\text {min }}(h)}\left(\frac{1}{r}\right)^{m}
$$

$$
\times \sum_{i=1}^{n}\left\{1+M_{i} \theta_{i}(h) \lambda_{j} \sum_{j=1}^{n}\left|a_{j i}^{*}\right| r^{\sigma_{j}+1} \sigma_{i}\right\} \times\left(\sup _{l \in[-\sigma, 0]_{z}}\left|\phi_{i}-\psi_{i}\right|\right),
$$

where

$$
\begin{aligned}
& \lambda_{\text {min }}=\min _{i=1,2, \cdots, n}\left\{\lambda_{i}\right\}, \lambda_{\max }=\max _{i=1,2, \cdots, n}\left\{\lambda_{i}\right\}, \\
& \theta_{\text {min }}(h)=\min _{i=1,2, \cdots, n}\left\{\theta_{i}(h)\right\}, \\
& \theta_{\text {max }}(h)=\max _{i=1,2, \cdots, n}\left\{\theta_{i}(h)\right\} .
\end{aligned}
$$

Therefore, we obtain

$$
\sum_{i=1}^{n}\left|x_{i}^{m}(\phi)-x_{i}^{m}(\psi)\right| \leq \alpha\left(\frac{1}{r}\right)^{m-\sigma} \sum_{i=1}^{n}\left(\sup _{l \in[-\sigma, 0]_{z}}\left|\phi_{i}-\psi_{i}\right|\right),
$$

for $m \in[-\sigma, \infty)_{Z}$,

where

$$
\begin{aligned}
\alpha & =\frac{\lambda_{\max } \theta_{\max }(h)}{\lambda_{\min } \theta_{\min }(h)} \max _{i=1,2, \cdots, n}\left\{1+M_{i} \theta_{i}(h) \sum_{j=1}^{n}\left|a_{j i}^{*}\right| r^{\sigma_{i}+1} \sigma_{i}\right\} \\
& \geq 1 .
\end{aligned}
$$

We can choose a positive integer $M$ such that

$$
\alpha\left(\frac{1}{r}\right)^{M \sigma-\sigma} \leq \frac{1}{4} \text {. }
$$

Define a Poincaré mapping $H: B \rightarrow B$ by $H \phi=x_{\sigma}(\phi)$. Then from (20), we can derive that

$$
\left\|H^{M} \phi-H^{M} \varphi\right\| \leq \frac{1}{4}\|\phi-\varphi\|,
$$

where $H^{M} \phi=x_{m \omega}(\phi)$. This implies that $H^{M}$ is a contraction mapping. Therefore, there exists a unique fixed point $\phi^{*} \in B$ such that $H^{M} \phi^{*}=\phi^{*}$. So, $H^{M}\left(H \phi^{*}\right)=H\left(H^{M} \phi^{*}\right)=H \phi^{*}$. This shows that $H \phi^{*} \in B$ is also a fixed point of $H^{M}$, hence, $H \phi^{*}=\phi^{*}$, that is, $x_{\omega}\left(\phi^{*}\right)=\phi^{*}$. Let $x\left(k, \phi^{*}\right)$ be the solution of (3) through $\left(0, \phi^{*}\right)$. By using $I(m+\varpi)=I(k)$ for $m \in Z^{+}, x\left(m+\varpi, \phi^{*}\right)$ is also a solution of (16). Note that $x_{m+\sigma}\left(\phi^{*}\right)=x_{m}\left(x_{\sigma}\left(\phi^{*}\right)\right)=x_{m}\left(\phi^{*}\right)$ for $m \in Z^{+}$, then $x\left(m+\varpi, \phi^{*}\right)=x\left(m, \phi^{*}\right)$ for $m \in Z^{+}$. This shows that $x\left(m, \phi^{*}\right)$ is a periodic solution of (3) with period $\varpi$. From (21), it is easy to see that all other solutions of (3) converge to this periodic solution exponentially as $m \rightarrow+\infty$.

Remark 2. When $I=\left(I_{1}, I_{2}, \cdots, I_{n}\right)$ is a constant vector, then for any constant $T \geq 0$ we have $I=I(m+T)=I(m)$ for $m \in Z^{+}$. Thus by the results obtained, when the sufficient condition in Theorem 2 is satisfied, a unique periodic solution becomes a periodic solution with any positive constants as its period. So, the periodic solution reduced to a constant solution, that is, an equilibrium point. Furthermore, all other solutions globally robustly exponentially converge to this equilibrium point as $m \rightarrow+\infty$. The unique equilibrium point of the discrete-time dynamical neural system (4) is globally exponentially stable. Obviously, the results obtained are consistent with the exponential stability results that were recently reported in (Mohamad and 
Gopalsamy, 2003), (Sun and Feng, 2004) and (Mahamad, 2001).

\section{CONCLUSIONS}

In this paper, robust exponential stability and periodicity of continuous-time and discrete-time dynamical systems for pattern recognition have been proposed. Without assuming the boundedness and differentiability of the activation functions, the easily checked conditions ensuring the exponential periodicity of attractor neural networks are obtained. It is shown that the discrete-time analogue preserves the dynamical characteristics of the continuous-time neural systems.

\section{REFERENCES}

Arik S., and Tavsanoglu V.(2003). Global robust stability of delayed neural networks. IEEE Transactions on Circuits and Systems I: Fundamental Theory and Applications, 50 (1), $156-160$.

Cao J. (2000). On exponential stability and periodic solutions of CNNs with delays, Physics Letters A, 267, 312-318.

Chen Y. H., and Fang S. C. (2000). Neurocomputing with time delay analysis for solving convex quadratic programming problems. IEEE Trans. Neural Networks, 11, 230-234.

Forti M., and Tesi A. (1995). New conditions for global stability of neural networks with application to linear and quadratic programming Problems, IEEE Trans. Circuits and Systems I, 42, 354-366.

Gopalsamy K., and He X. Z. (1994). Delayindependent stability in bi-directional associative memory networks. IEEE Trans. Neural Networks, 5, 998-1002.

Kennedy M., and Chua L. (1988). Neural networks for linear and nonlinear programming, IEEE Trans. Circuits \& Systems, 35, 554-562.

Liao X. F., and Yu J. B.(1998). Robust interval stability analysis of Hopfield neural network with time delay. IEEE Tans Neural Networks, 9(5), 1042-1045.

Liao X. X., and Wang J.(2003). Algebraic criteria for global exponential stability of cellular neural networks with multiple time delays. IEEE Trans. Circuits Systems I, 50 (2), 268-275.

Liu D. R., and Lu Z. (1997). A new synthesis approach for feedback neural networks based on the percetron training algorithm. IEEE Trans. Neural Networks, 8,1468-1482.

Mohamad S. (2001). Global exponential stability in continuous-time and discrete-time delay bidirectional neural networks, Physical D, 159, 233-251.
Mohamad S., and Gopalsamy K. (2003). Exponential stability of continuous-time and discrete-time cellular neural networks with delays. Applied Mathematics and Computation, 135, 17-38.

Sudharsanan S., and Sundareshan M. (1991). Exponential stability and a systematic synthesis of a neural network for quadratic minimization. Neural Networks, 4, 599-613.

Sun C., et al. (2002). On Exponential Stability of Delayed Neural Networks with a General Class of Activation Functions. Physics Letters A. 298 (2/3), 122-132.

Sun C., and Feng C. B. (2005). Discrete-time analogues of integrodifferential equations modeling neural networks. Physics Letters A, 334, 180-191.

Sun C., and Feng C. B. (2004). Exponential periodicity and stability of delayed neural networks. Mathematics and Computers in Simulation, 66, 469-478.

Sun C., and Feng C. B. (2004). Exponential periodicity of continuous-time and discrete-time neural networks with delays. Neural Processing Letters, 19(2):131-146.

Tank D. W., and Hopfield J. J. (1986). Simple "neural" optimization networks: An A/D converter, signal decision circuit, and a linear programming circuit, IEEE Trans. Circuits \& Systems, 33, 533-541.

Townley S. (2000). Existence and learning of oscillations in recurrent neural networks. IEEE Trans. Neural Networks, 11, 205-214.

Van Den Driessche P. and Zou X. (1998). Global attactivity in delayed Hopfield neural network models, SIAM Journal of Applied Mathematics, $58,1878-1890$.

Venetianer P. L., and Roska T. (1998). Image compression by delayed CNNs. IEEE Trans. Circuits Syst. I. 45, 205-215.

Zhang Y., Pheng H. A., and Vadakkepat P.(2002). Absolute periodicity and absolute stability of delayed neural networks. IEEE Trans. Circuits and Systems: I, 49, 256-261. 